\title{
An Interactive Application for Removal of Bone Information in CT-Angiography
}

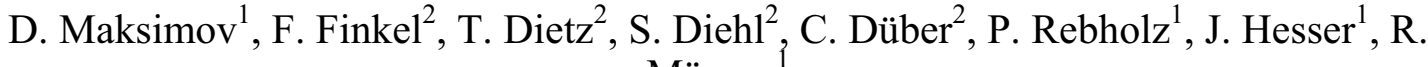 \\ Männer ${ }^{1}$ \\ ${ }^{1}$ ICM, Universities of Mannheim and Heidelberg, Mannheim, Germany \\ ${ }^{2}$ Dept. of Radiology, University of Heidelberg, Mannheim, Germany \\ maksimov@ti.uni-mannheim.de
}

\begin{abstract}
In this paper we present a method that allows removing bony structures and calcifications from CT angiography (CTA) data. It relies on two data sets, one with (CA) and one without (NCA) contrast agent. In contrast to current approaches, the algorithm uses local rigid matching of each single object. Therefore, it allows compensating motion of the patient during acquisition. Furthermore, the method uses a highly accurate surface-matching procedure so that local mismatches can be as small as 3 voxels and therefore even arteries near the bone are mostly separated correctly.

The overall system requires about 20 minutes on a Pentium $42.4 \mathrm{GHz}$ with 1 GByte RAM for a 700 MByte data set (CA+NCA) and is currently used in the clinic for evaluation purposes.
\end{abstract}

\section{Introduction}

For the diagnosis of arterial stenosis the former golden standard the digital subtraction angiography (DSA) has been replaced by either Doppler resp. duplex ultrasound or MR angiography [16,17]. However, for cases like ferro-magnetic metal implants or claustrophobia, there is a demand for an alternative solution. With upcoming multi-detector CTs there exists a solution, i.e. CT angiography (CTA). It further offers both the visualization of contrast agent and calcification in 3D. Its disadvantage is currently that the arteries are overlaid by bright objects like bone and calcifications [10-15].

\section{State of the art}

In this paper we address the problem of fast and accurate removal of bone information from the CTA images so that this tool can be used in daily practice. There have been different groups tackling this problem as well [1-9]. For example, Straka et al. [1,2] combine thresholding and anatomic atlases for eliminating bone structures. Their automatic method requires in the worst case 45 minutes for that procedure and doesn't require an additional volume without contrast agent. Neumann et al. [18] mask bones in a data set without contrast agent, register it with a data set with contrast agent and remove the bony structures. The LiveWire algorithm from Knapp et al.[4] consists of two basic steps. Firstly, contours are outlined by the user on a small number of planar cross-sections through the object using live-wire. Secondly, the traced contours are used for reconstructing the object surface automatically in each slice again by live-wire.

Other techniques detect vessels directly and then separate them from bones. Zahlten et al.[5,6] use region growing and bifurcation detection for finding vessels where the initial seedpoint is set manually. Falcao et al.[7,8] developed a three stage algorithm. The dataset is preprocessed by a 3D vesselness operator, tubular structures are tracked with a minimal cost path through the centerline of the vessels and finally, vessel borders are determined. 
All these methods, however, have in common that vessels near the bone are not separated correctly, i.e. the volume of the vessel is either under- or overestimated. In addition, no method currently is able to remove calcifications from the vessel data. These two problems are the issue of our approach.

\section{Method}

Our goal is an automatic and fast alternative for bone removal for CTAs; which can partially be applied for calcifications as well.

When we started the project, we initially assumed that both the positional accuracy of CTs is below $1 \mathrm{~mm}$ and the patient can be fixated so that we can neglect any motion. Two data sets are acquired, one with (CA) and one without (NCA) contrast agent. Bone and calcification are segmented by thresholding (about 200HU) in the NCA data and mask out the corresponding voxels in the CA data set. However, we quickly found out that both preconditions are not fulfilled. The repetition accuracy of CTs is often $>1 \mathrm{~mm}$ and only a few patients could be fixated tightly. Often, the lower extremities even moved during acquisition.

As a remedy, we developed a locally rigid, globally non-rigid matching approach, which is described in the following.

First of all, all objects in the NCA and the CA data set that are above a threshold (about 200HU) are segmented. We differentiate between bone and calcification using a size criterion. Objects consisting of more than 200 pixels for pelvis and 50 pixels for extremities on each slice are considered as bone whereas objects below that threshold are assigned to calcifications. The selection of this size is, however, problem dependent. For patients with huge calcified regions, the threshold must be higher than for others. Therefore, this parameter can be determined during the analysis phase.

Next, we assume that the patient moves only slowly (otherwise each acquisition is blurred and a detailed analysis would not be possible). Therefore, we can assume that for a short time of the acquisition, we can neglect any motion. Each bony compartment should therefore have the same shape in both data sets if considering only a few slices. We selected 10 slices as a compromise between accuracy of matching and accuracy of the shape information.

The data volume is therefore analyzed in overlapping stacks of 10 consecutive slices. In each such stack different objects (bones/calcification objects) are labeled as a different object. An assignment between objects in NCA and CA is based on their relative position in the data sets. This assignment is a good choice since we assume only little motion even between both data sets and misassignments have not been observed during the current study.

In the matching step, we applied the following method to each of such pairs: Firstly, we extract the surface of both objects. Next, we calculate a 3D distance data for the CA-data that describes for each voxel in CA the distance to the nearest surface. Iterative closest point surface matching was used to match both objects where the distance coding allows to calculate the error measure with $\mathrm{O}(1)$ operations. More details about this method are described in [9].

After matching all objects of the NCA-image are resampled in the CA-image coordinate system and then are considered as a mask. Only those objects in the CA-image are eliminated that were classified as bone. The elimination uses the aforementioned mask to set the voxel values to non-visible. Since there are still subtle matching and segmentation inaccuracies (partial volume effect), the mask is locally modified by at most by 3 pixels in order to adapt it to the underlying segmented structure. It is extended when not completely covering neighboring bone information in the CA-data set and shrinked when covering non-classified 
structures. For calcification this local adaption of the shape is suppressed since it is difficult to differentiate between calcification and contrast agent - otherwise the cross-section of the artery would be severely underestimated.

In the final step, we perform a masking of the unwanted structures. We use the VGLlibrary of Volume Graphics $\mathrm{GmbH}$ that allows setting segmentation bits which allow rendering marked objects as transparent. Rendering is then based on the standard MIP.

\section{Results}

The overall procedure takes currently 20 minutes for a 700 MByte data set $(512 \times 512$ pixels per slice, approx. 700 slices, both data sets, CA and NCA) and a Pentium 2.4 PC with 1 GByte of RAM. Fig. 1 shows a typical result and fig. 2 gives a close-up of the same data. As can be seen, the bone information is fully removed. In the knee area there are some regions below the threshold where, due to the partial volume effect, there are still some voxels that partially represent bony structures but they are less in contrast than the contrast agent. Therefore, they are not relevant for the practical use.
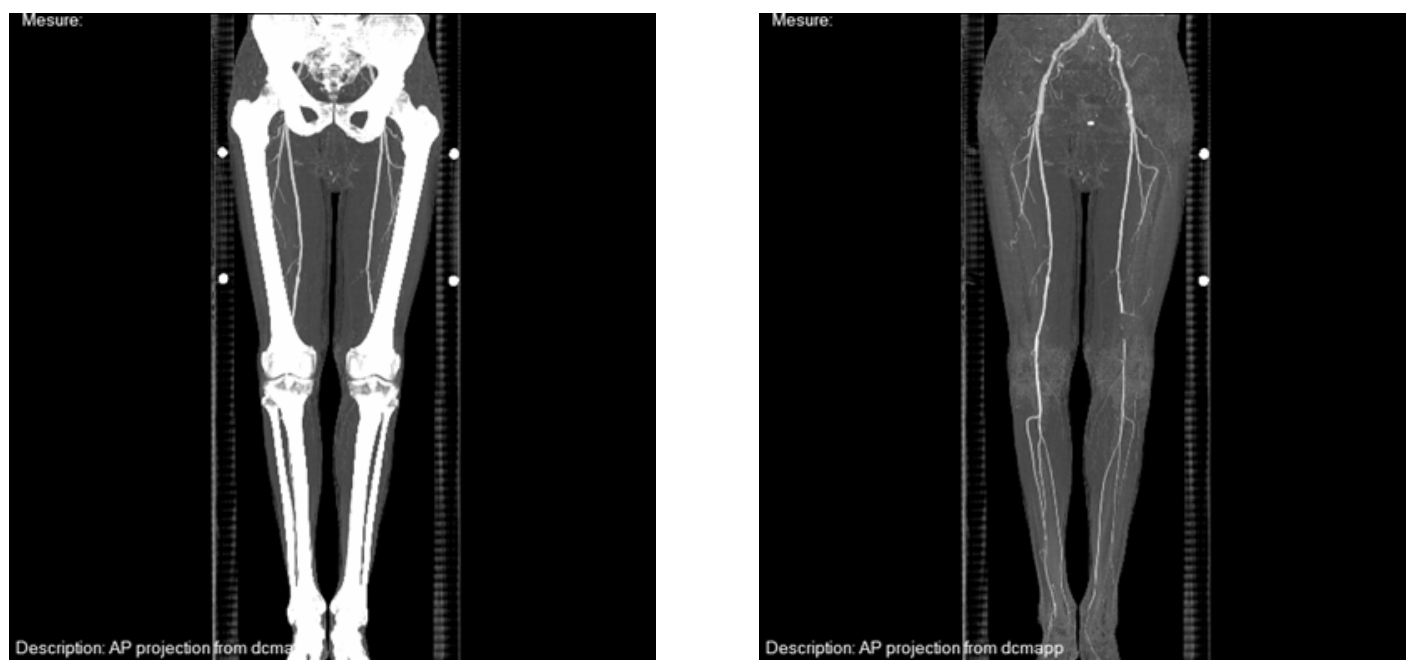

Figure 1. Left: MIP image of CA-image of a patient, right: MIP of the same data set when bones have been removed.
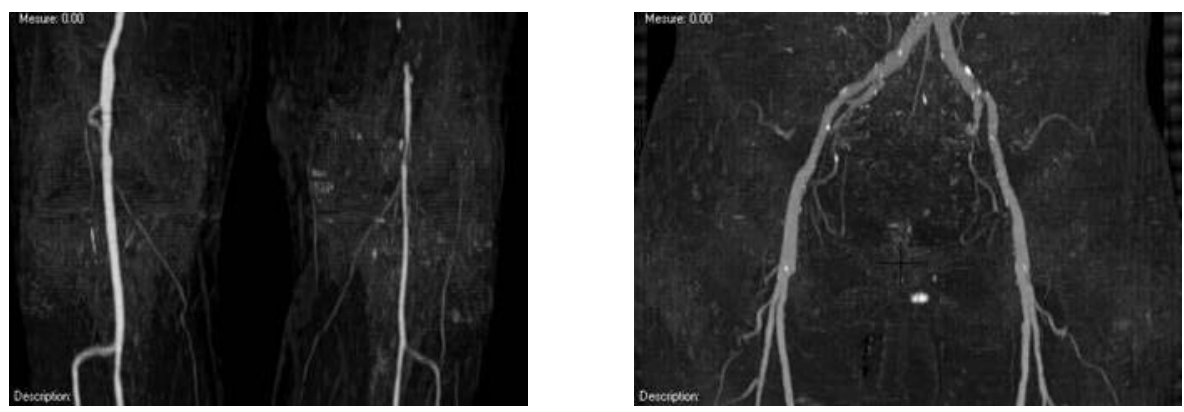

Figure 2. Close-ups of a critical region near the knee, left, (where severe movements occurred) and the hip, right, where large calcified regions could be found.

Another limitation of our approach is the incorrect removal of calcifications in some cases. Such a case is illustrated in fig. 3. Fig. 4 shows that there was a shift of the soft tissue that is the reason for this sort of error. This indicates that for a correct operation, 
elastic matching in small scales is relevant as well. Fig. 5 shows a case where small matching inaccuracies don't mask out the calcification fully.

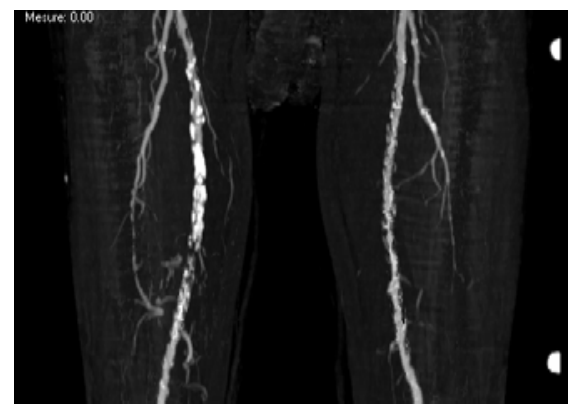

Figure 3. Left leg: incorrect removal of calcification, right leg: correct removal.
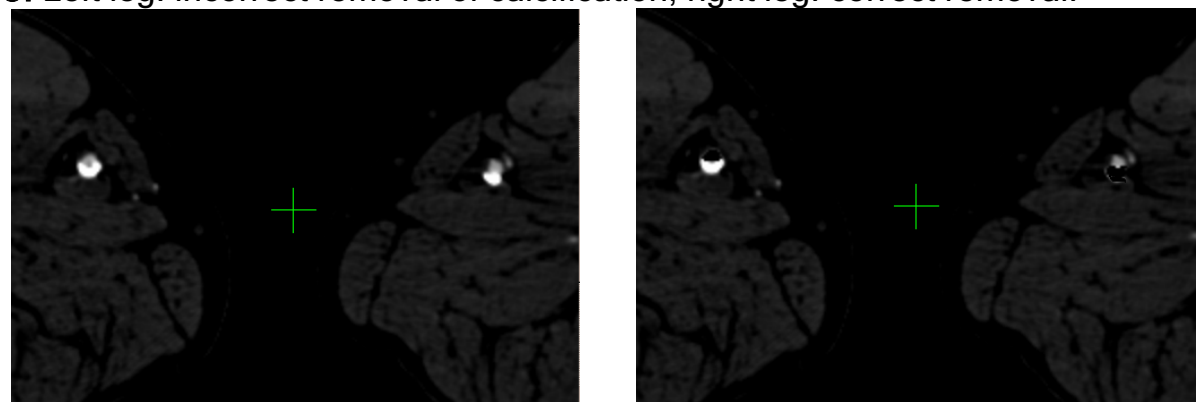

Figure 4. Slice information for the case shown in fig. 3 before (left) and after (right) removal of calcification.
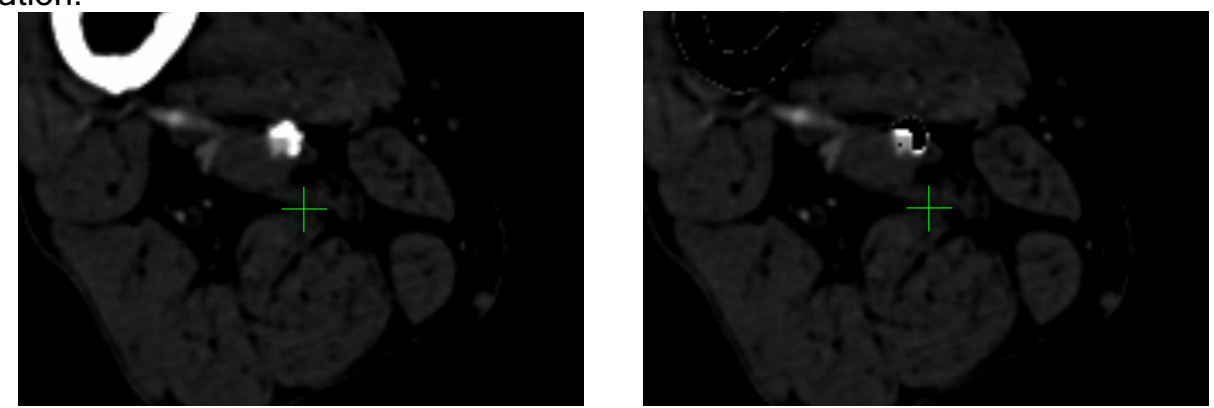

Figure 5. Slice information before (left) and after (right) removal of calcification where the calcification is not fully masked out due to small matching inaccuracies.

Fig. 6 shows a case where the artery is too near to the bone structure so that part of the artery is removed as well. Here, the calcification is considered as part of the bone by the algorithm since both parts are not separated by low-intensity voxels. Fig. 7 illustrates the case where a small vessel is erroneously removed together with bone since it is adjacent to it.
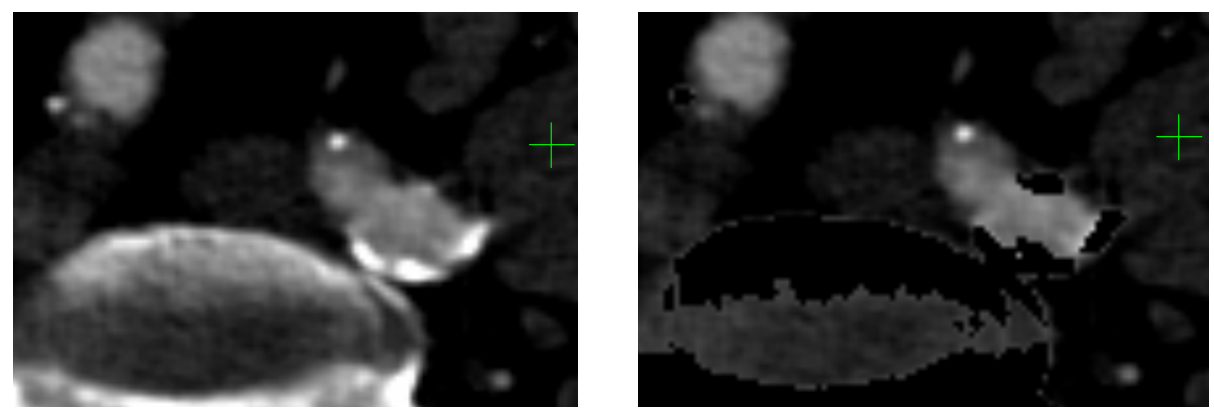

Figure 6. Slice information before (left) and after (right) removal of calcification where contrast-agent-regions are masked out since the artery is too near to the bone. 

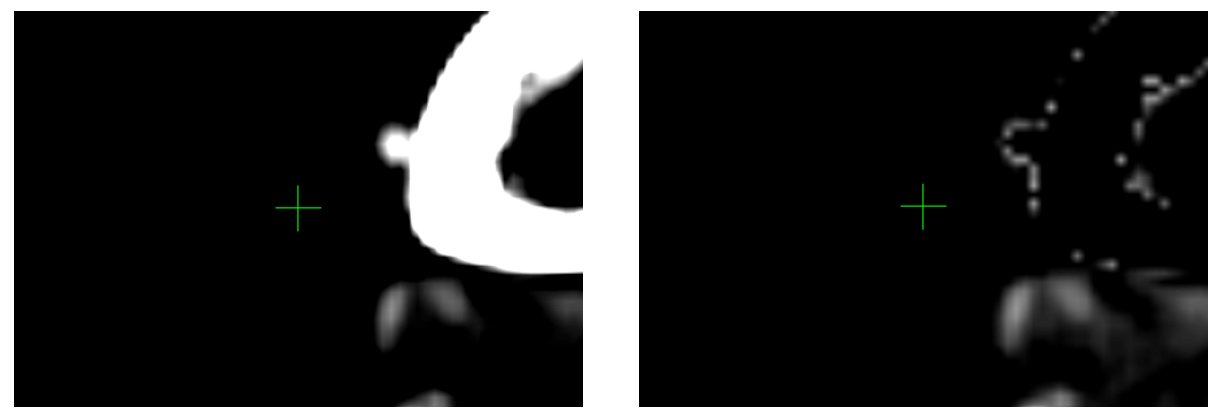

Figure 7. Slice information before (left) and after (right) removal of calcification. The vessel is too near to the bone and removed.

\section{Conclusions and Outlook}

In this paper we have presented a method that allows remove bony structures as well as calcifications in CT angiographic data using local matching and image processing. The method gives robust results in most cases so that a diagnosis of the degree of stenosis is possible for arteries of diameter $>2 \mathrm{~mm}$. Nevertheless, the method has shortcomings in the case where arteries are near the bone and second, it underestimates the cross-section of arteries in the presence of calcifications due to the accuracy limitations of rigid matching.

We intend to solve both problems with better non-rigid matching strategies and by problem specific object segmentation methods.

\section{References}

[1] M. Straka, A. La Cruz, A. Köchl, M. Sramek, E. Gröller, and D. Fleischmann, "3D Watershed Transform Combined with a Probabilistic Atlas for Medical Image Segmentation", Proceedings of MIT2003, 2003.

[2] M. Straka, A. La Cruz, L.I. Dimitrov, M. Sramek, D. Fleischmann, and E. Gröller, "Bone Segmentation in CTAngiography Data Using a Probabilistic Atlas", Proceedings of Vision Modeling and Visualization, 2003, pp. 505512.

[3] A.M. Alyassin, and G.B. Avinash, "Semi-automatic bone removal technique from CT angiography data", Proceedings of SPIE Vol. 4322, 2001, pp. 146-146.

[4] M. Knapp, A. Kanitsar, and M.E. Gröller, "Semi-Automatic Topology Independent Contour-Based 2² D Segmentation Using Live-Wire", Proceedings of WSCG 2004, 2004.

[5] C. Zahlten, H. Jürgens, H. O. Peitgen, C.J.G. Evertsz, R. Leppek, and K.J. Klose, "Portal vein reconstruction based on topology", Radiology, 1993. http://www.cevis.uni-bremen.de/article-db/artikel/conni/EuropRadiol.ps.

[6] C. Zahlten, "Reconstruction of branching blood vessels from CT-data", In B.Urban M. Göbel, H. Müller, editor, Visualization in Scientific Computing, Springer-Verlag, Wien, (1995), pp. 41-52.. http://www.cevis.unibremen.de/artdb/artikel/conni/Rostock.ps.

[7] A.X. Falcao, J.K. Udupa, S. Samarasekera, S. Sharma, B.E. Hirsch, and R.A. Lotufo, "User-steered image segmentation", Graphical models and image processing, 1998, pp. 233-260.

[8] A.X. Falcao, J.K. Udupa, and F.K. Miyazawa, "An ultra-fast user-steered image segmentation paradigm: Live wire on the fly”, IEEE Transaction on Medical Imaging, January 2000, pp. 55-62.

[9] O. Krivonos, P. Keppler, F. Gebhard, L. Kinzl, J. Hesser, R. Männer, "From Planning of Complex Bone Deformities Correction to Computer Aided Operation", In 14th IEEE Symposium on Computer-Based Medical Systems, 26-27 July 2001, pp. 210-215.

[10] G.D. Rubin, A.J. Schmidt, L.J. Logan, and M.C. Sofilos, "Multi-Detector Row CT Angiography of Lower Extremity Arterial Inflow and Runoff: Initial Experience”, Radiology 2001, pp. 221: 146-158.

[11] H.W. Venema, F.J.H. Hulsmans, and G.J. Heeten, "CT Angiography of the Circle of Willis and Intracranial Maxiumum Intensity Projection with Matched Mask Bone Elimination - Feasibility Study”, Radiology 2001, pp. 218:893-898. 
[12] M.L. Martin, K.H. Tay, B. Flak, P.D. Fry, D.L. Doyle, D.C. Taylor, Y.N. Hsiang, and L.S. Machan, "Multidetector CT Angiography of the Aortoiliac System and Lower Extremities", AJR 2003, pp. 180: 1085 1091.

[13] A. Ofer, S.S. Nitecki, S. Linn, M. Epelman, D. Fischer, T. Karram, D. Litmanovich, H. Schwartz, A. Hoffman, and A. Engel, "Multidetector CT Angiography of Peripheral Vascular Disease", AJR 2003, pp. 180:719 $-724$.

[14] H. Ota, K. Takase, K. Igarashi, Y. Chiba, K. Haga, H. Saito, and S. Takahashi, "MDCT Compared with Digital Substraction Angiography for Assessment of Lower Extremeity Arterial Occlusive Disease", AJR 2004, pp. 182: 201-209.

[15] B. Tins, J. Oxtoby, S. Patel, "Comparison of CT angiography with conventional arterial angiography in aortoiliac occlusive disease", Br J Radiology 2001, pp. 74: 219 - 225.

[16] H.P. Busch, H.G. Hoffmann, J. Rock, and C. Schneider, "MR angiography of pelvic and leg vessels with automatic table movement technique ("Mobi-Trak")--clinical experience with 450 studies", Rofo Fortschr Geb Rontgenstr Neuen Bildgeb Verfahr, 2001 May.

[17] A. Hentsch, et al., "Gadobutrol-enhanced moving-table magnetic resonance angiography in patients with peripheral vascular disease: a prospective, multi-centre blinded comparison with digital subtraction angiography“, Eur. Radiology, 2003.

[18] J. Neumann, Z. Lin, P. Rösch, C. Lu, J. Lu, R. Ma, M. Steinmiller, D. Smith, and J. Durgan, "Clinical Evaluation of a Registration-Based Bone Masking Technique for Improved CT Angiography", European Congress of Radiology, 2003. 